\title{
Redução de vigor em pereiras europeias utilizando métodos físicos e químicos
}

\author{
Vegetative growth control in European pear trees through physical and chemical methods
}

\author{
Alberto Ramos Luz ${ }^{1 *}$, Deivid Silva de Souza ${ }^{2}$, Rafael Petineli ${ }^{3}$, Joseane de Souza Hipólito ${ }^{4}$, Leo \\ Rufato $^{2}$ \& Aike Anneliese Kretzschmar ${ }^{2}$ \\ ${ }^{1}$ Profissional Autônomo, Antônio Prado, RS, Brasil. *Autor para correspondência: albertoramosluz@yahoo.com.br. \\ ${ }^{2}$ Universidade do Estado de Santa Catarina, Lages, SC, Brasil. \\ ${ }^{3}$ Universidade Estadual de Londrina, Londrina, SC, Brasil. \\ ${ }^{4}$ Profissional autônomo, Criciúma, SC, Brasil.
}

Submissão: 24/11/2016 / Aceite: 09/05/2018

\begin{abstract}
RESUMO
As diversas tentativas falhas de produção de peras no Brasil são impulsionadas pelo elevado consumo, que apresenta um cenário crescente de importação para atender a demanda interna. Em 2015 foram importadas 179 mil toneladas de peras, sendo a maior parte da Argentina e Portugal. A limitação do cultivo de pereira não tem sido por falta de mercado, mas devido a diversos fatores, como por exemplo, a baixa frutificação efetiva, a insuficiente formação de estruturas de produção e o excesso de crescimento vegetativo. O objetivo deste trabalho foi avaliar o crescimento vegetativo e frutificação efetiva de pereiras europeias submetidas a diferentes técnicas de redução de vigor. $O$ experimento foi conduzido na safra 2012/2013 e 2013/2014 em um pomar comercial de pereiras localizado no município de Vacaria, RS, situada a $971 \mathrm{~m}$ de altitude. As pereiras Packham's Triumph, William's, Red Bartlett, Abate Fetel e Carrick, todas enxertadas sobre o portaenxerto Pyrus calleryana, foram submetidas aos tratamentos de anelamento simples, anelamento duplo, corte de tronco simples, corte de tronco duplo e aplicação do fitorregulador Trinexapac-etil (1600 $\left.\mathrm{mg} \mathrm{L}^{-1}\right)$. De acordo com os resultados obtidos foi possível observar que houve redução do crescimento vegetativo apenas na cultivar Packham's submetida ao anelamento duplo, corte de tronco simples e Trinexapac-etil. A utilização da técnica de anelamento e corte de tronco incrementa o retorno de floração e também a produtividade das pereiras 'Abate Fetel' e 'Carrick', bem como a aplicação de Trinexapac-etil reduz o retorno de florescimento destas cultivares nas condições do estudo.
\end{abstract}

PALAVRAS-CHAVE: Pyrus communis L., reguladores de crescimento, anelamento, corte do tronco, frutificação efetiva.

\section{ABSTRACT}

The various failed attempts to produce pears in Brazil are driven by high consumption, which presents a growing scenario of imports to attend to the consumers demand. In 2015, 179 thousand tons of pears were imported, most from Argentina and Portugal. The limitation of pear cultivation has not been due to lack of market, but due to several factors, such as low fruit set, insufficient formation of production structures and excessive vegetative growth. The objective of this work was to evaluate the vegetative growth and fruit set of European pear trees submitted to different growth control techniques. The experiment was conducted during 2012/2013 and 2013/2014 season in a commercial pear orchard located in Vacaria city, Rio Grande do Sul State, at 971 m altitude. Packham's Triumph, William's, Red Bartlett, Abate Fetel and Carrick, all grafted on Pyrus calleryana, were submitted to treatments of simple girdling, double girdling, single trunk cutting, double trunk cutting and application of the Trinexapac-ethyl plant growth regulator $\left(1600 \mathrm{mg} \mathrm{L}^{-1}\right)$. According to the results, it was possible to observe that there was a reduction of vegetative growth only in the Packham's cultivar submitted to double girdling, single trunk cutting and Trinexapac-ethyl. The use of the trunk cutting and girdling technique increases the flowering return and also the productivity of 'Abate Fetel' and 'Carrick' pear trees, and the Trinexapac-ethyl decreases the return bloom of 'Abate Fetel' and 'Carrick' pear trees in the studied conditions.

KEYWORDS: Pyrus communis L., plant growth regulators, trunk cutting, fruit set, girdling. 


\section{INTRODUÇÃO}

A pereira pertence à família Rosaceae, subfamília Pomoideae e gênero Pyrus. Compreende mais de 20 espécies, todas nativas da Europa e da Ásia, sendo as mais importantes pertencentes às espécies: Pyrus communis (Europeia), P. pyrifolia (Japonesa), P. bretschneideri (Chinesa) e híbridos entre $P$. communis e P. pyrifolia (QUEZADA et al. 2003).

O crescimento vegetativo das pereiras é responsável pelo acúmulo de carboidratos gerados através da fotossíntese. A partição destes carboidratos nas partes vegetativa e reprodutiva da planta determina a produção dos pomares (JACKSON 2003). Segundo IUCHI \& LUZ (2010), o excessivo crescimento vegetativo tem efeito negativo sobre a formação de gemas florais, principalmente por sombrear a parte interior e inferior do dossel, confirmando o antagonismo entre as partes vegetativas e reprodutivas das pereiras.

Para contornar o excesso de vigor das pereiras enxertadas sobre portaenxertos vigorosos, algumas técnicas de manejo, tanto físicas como químicas podem ser utilizadas, a exemplo do anelamento de tronco, corte de tronco e aplicação de fitorreguladores.

As técnicas de anelamento e corte de tronco consistem na retirada de parte dos tecidos vasculares, diminuindo o aporte de carboidratos para a raiz e de água e nutrientes para a copa. O corte de tronco com motosserra é realizado com o objetivo de reduzir o crescimento vegetativo das árvores e melhorar a regularidade de produção das culturas. No entanto, os resultados destas medidas são difíceis de prever e podem reduzir a qualidade do fruto no momento da colheita ou durante o armazenamento (DECKERS et al. 2005). IUCHI et al. (2008), em trabalhos desenvolvidos no sul do Brasil, descreveram redução do crescimento vegetativo da pereira 'Packham's Triumph' enxertada sobre $P$. calleryana seguido de aumento de produtividade ocasionados pela utilização da técnica de anelamento.

Prohexadiona-Ca ( $\mathrm{PCa}$ ) e Trinexapac-etil (Trin-etil) são reguladores de crescimento introduzidos no mercado mundial desde a década de 90 , sendo utilizados na agricultura, horticultura e viticultura. Apesar da sua semelhança química, apresentam diferenças no modo de ação. Trin-etil é quase inteiramente usado em espécies gramináceas, enquanto que $\mathrm{PCa}$ é também utilizado em frutíferas e outras espécies de dicotiledóneas (RADEMACHER 2014).

No Brasil, a utilização de PCa em frutíferas é conhecido, enquanto que o Trin-etil é utilizado apenas em cana-de-açúcar e cereais, onde atua regulando o crescimento do colmo por meio da redução do elongamento do internódio promovido pela inibição da biossíntese de giberelinas, provocando uma inibição temporária ou redução do ritmo de crescimento, sem afetar, porém, o processo de fotossíntese e a integridade da gema apical.

Embora a utilização de formas naturais para controle de crescimento vegetativo seja mais recomendada, a utilização de reguladores de crescimento químicos apresenta-se como boa alternativa quando o pomar encontra-se completamente fora de equilíbrio vegeto-produtivo, pois é necessário buscar novas soluções para o manejo da cultura, além da redução dos custos com mão de obra. Contudo, a aplicação de fitorreguladores em pereiras está em discussão a nível mundial, porque alguns destes podem influenciar negativamente sobre o retorno de floração (DECKERS et al. 2005).

Diante deste contexto, objetivou-se estudar o efeito do anelamento e corte de tronco, bem como a aplicação de Trinexapac-etil sobre o comportamento vegetativo e produtivo de pereiras europeias.

\section{MATERIAL E MÉTODOS}

O experimento foi conduzido na safra 2012/2013 e 2013/2014 em um pomar comercial de pereiras localizado no município de Vacaria, RS, situada a $971 \mathrm{~m}$ de altitude, com coordenadas geográficas de 2830'44" latitude Sul e 5056'02" longitude Oeste.

Foram utilizadas pereiras adultas (plantio 1988), das cultivares Packham's Triumph, William's, Red Bartlett, Abate Fetel e Carrick, todas enxertadas sobre Pyrus calleryana, com espaçamento de $5 \mathrm{~m}$ entre filas e $2 \mathrm{~m}$ entre plantas, totalizando 1.000 plantas por hectare. As plantas encontravam-se conduzidas em líder central sem tutoramento.

Os tratamentos utilizados foram: Anelamento simples; anelamento duplo (Figura 1); corte de tronco simples (um corte até a metade do diâmetro do tronco); corte de tronco duplo (dois cortes de 1/3 do diâmetro do tronco, realizados em posições opostas) (Figura 1); e aplicação do fitorregulador Trinexapac-etil (1600 $\mathrm{mg} \mathrm{L}^{-1}$ ).

O anelamento foi realizado no início do florescimento de cada cultivar, nas duas safras em estudo, com o auxílio de um anelador, o qual retirava um anel de casca, em torno de toda a circunferência do tronco, de aproximadamente $4 \mathrm{~mm}$. 
Para a realização do corte de tronco, este realizado apenas no primeiro ano, utilizou-se motosserra com óleo vegetal para lubrificação da correia, utilizou-se ainda espuma de poliuretano para vedar o corte, com objetivo de impedir a infecção por doenças. Trin-etil foi aplicado nas duas safras em estudo, em torno de duas semanas após a plena floração, quando os brotos se encontravam de 5 a $10 \mathrm{~cm}$ de comprimento. Para isto, preparou-se as soluções momentos antes de serem utilizadas, sendo aplicadas uma única vez em cada planta, com o auxílio de pulverizador costal motorizado, com volume de calda equivalente a $1000 \mathrm{~L}$ ha $^{-1}$.

(A)

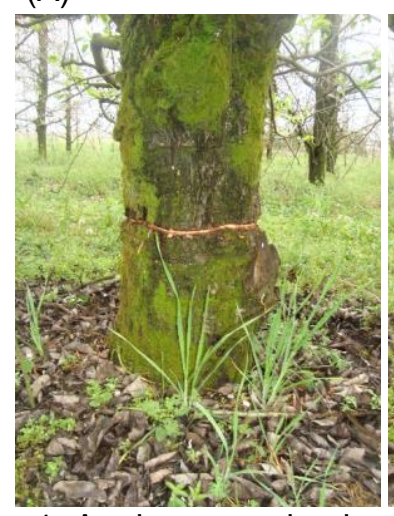

(B)

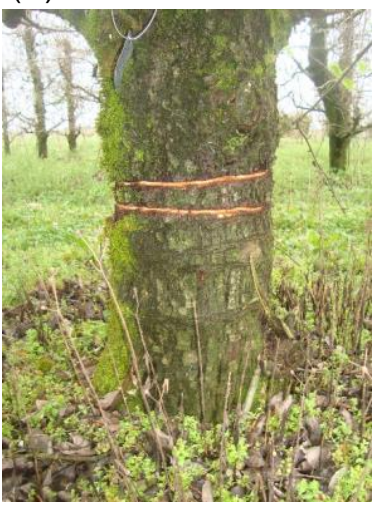

(C)

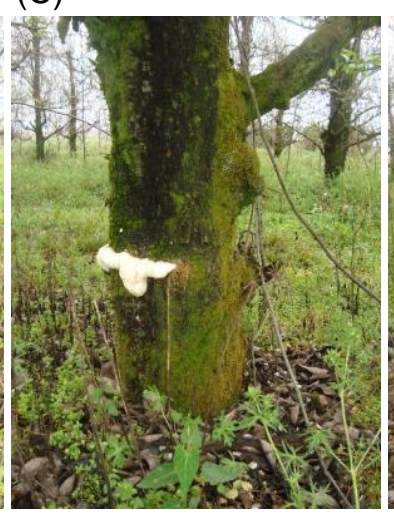

(D)

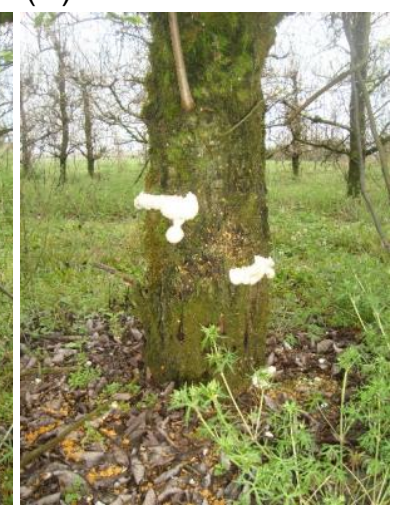

Figura 1. Anelamento simples (A), anelamento duplo (B), corte de tronco simples (C) e corte de tronco duplo (D) realizado em pereira 'Packham's Triumph' no município de Vacaria, RS, durante o início de floração da safra 2012/2013.

Figure 1. Single girdling $(A)$, double girdling $(B)$, single trunk cut $(C)$ and double trunk cut $(D)$ performed in 'Packham's Triumph' pear tree in Vacaria city, RS, Brazil during the beginning of flowering of 2012/2013 season.

As variáveis avaliadas foram:

- Frutificação efetiva: efetuou-se a contagem do número total de flores em quatro ramos por planta, e 60 dias após a plena floração, realizou-se a contagem das frutas remanescentes. A frutificação efetiva, expressa em porcentagem, foi determinada através da relação ( $n^{\circ}$ de frutos $\times 100$ ) / $n^{\circ}$ flores.

- Retorno de floração: efetuou-se a contagem do número total de flores em uma amostra de quatro ramos secundários por planta, escolhidos de forma a padronizar a posição, o comprimento e o vigor do ramo, com o valor multiplicado pelo número de ramos laterais por planta, tornando possível a estimativa do número de flores por planta.

- Produção por planta: colheita individual de cada planta em um único repasse, com posterior contagem e pesagem dos frutos colhidos, para permitir determinar a produção individual de cada planta.

- Produtividade estimada: uma vez obtida a produção por planta, estimou-se a produtividade através da multiplicação do número de plantas existentes em um hectare pela produção por planta, onde os dados foram expressos em toneladas ha-1.

- Diâmetro de frutos: com o auxílio de um paquímetro digital, mensurou-se o diâmetro de todos os frutos de cada planta amostrada, na qual se mediu a porção de maior diâmetro em lados opostos, a fim de se obter a porcentagem de frutos nas classes menor que $65 \mathrm{~mm}$, de $65 \mathrm{~mm}$ a $80 \mathrm{~mm}$ e maior que $80 \mathrm{~mm}$.

- Firmeza de polpa: foi determinada com o auxílio de um penetrômetro manual com ponteira de $8 \mathrm{~mm}$, acoplado a um suporte de aço. Para efetuar a leitura, efetuaram-se dois cortes para retirada da casca dos frutos, em lados opostos, na zona equatorial dos frutos, inserindo-se posteriormente a ponteira do penetrômetro na polpa da fruta, com os dados expressos em libras $\mathrm{cm}^{-2}$. Foram utilizadas amostras de 20 frutos por parcela, colhidos aleatoriamente.

- Sólidos solúveis: foi determinado utilizando o suco de 20 frutos por parcela, colhidos aleatoriamente, com o auxílio de um refratômetro digital de bancada (modelo RTD-45).

- Acidez titulável: foi determinada por titulação manual com $\mathrm{NaOH}$ a $0,1 \mathrm{~N}$. A solução foi preparada com grupos de 20 frutos por parcela (sobre uma solução de $5 \mathrm{ml}$ de suco, ao qual foi adicionado $5 \mathrm{ml}$ de água destilada e 3 gotas de azul de bromotimol) até atingir o ponto de viragem, os dados foram expressos em meq L-1.

- Crescimento anual da copa $(\mathrm{m})$ : avaliado durante o período de repouso vegetativo, calculado através da multiplicação do crescimento médio dos ramos do ano (amostrados 20 ramos do ano por planta) pelo número de brotações de ramos do ano (contou-se o número total de ramos do ano em uma amostra de 
quatro ramos secundários por planta, e contou-se também o número de ramos secundários por planta, possibilitando estimar a quantidade de ramos do ano por planta).

O delineamento experimental utilizado foi em blocos casualizados, com seis blocos e três plantas por parcela, onde avaliou-se a planta central e as demais utilizaram-se como bordadura. Os dados obtidos foram submetidos à análise de variância e as variáveis com diferenças significativas foram comparadas pelo teste de Scott-knott, à $5 \%$ de probabilidade de erro, através do programa computacional Sisvar versão 5.3 (FERREIRA 2010). Os dados em porcentagem foram transformados para arco seno da raiz quadrada do valor expresso em porcentagem dividido por $100(\operatorname{arcoseno}(\sqrt{ } \mathrm{x})) / 100$.

\section{RESULTADOS E DISCUSSÃO}

$\mathrm{Na}$ Tabela 1, pode-se observar que não houve efeito dos tratamentos sobre a frutificação efetiva das cultivares em estudo, durante a safra 2012/2013. As cultivares Abate Fetel e Carrick apresentaram baixa frutificação efetiva em todos os tratamentos, em média 1 e $3 \%$, respectivamente, o que demonstra que houve algum problema no processo de frutificação que refletiu em baixas produtividades (Tabela 1). De acordo com LUZ et al. (2012), são inúmeros os fatores que podem prejudicar o processo de frutificação efetiva em pereiras, como ausência de insetos polinizadores, temperatura desfavorável à polinização e fecundação, chuva durante o florescimento, ausência de cultivares polinizadores, incompatibilidade de polinização ou não coincidência de floração entre cultivar produtora e polinizadora, longa distância a ser percorrido pelo pólen entre sua origem e a flor de destino, entre outros. Já as cultivares Packham's Triumph, William's e Red Bartlett apresentaram em média $10 \%$ de frutificação efetiva, o que, segundo VAN DER ZWET \& CHILDERS (1982), é uma boa taxa para atingir elevadas produções em pereiras cuja floração é abundante, enquanto que pereiras com pouca floração necessitam de frutificação efetiva superior à $30 \%$. SMIT et al. (2005) não obtiveram efeito sobre a frutificação das cultivares de pereiras Rosemarie, Forelle, Packham's Triumph e William's submetidas à anelamento no período entre plena floração e queda de pétalas, durante dois anos consecutivos.

A utilização de métodos para redução de crescimento vegetativo em pereiras geralmente não altera a produtividade no primeiro ano de sua execução, já que suas estruturas reprodutivas ocorrem sobre ramos de ano ou de 2 anos, sendo, portanto, diferenciadas no ciclo anterior. No entanto, efeitos da redução de vigor sobre a produção podem ocorrer nos ciclos seguintes, pois a partição de fotoassimilados tende a melhorar através da eliminação de drenos vegetativos, tais como ramos ladrões, e do aumento da interceptação de luz no interior da planta, tornando possível a formação de estruturas reprodutivas em partes anteriormente sombreadas. De acordo com CAIN (1971) e DEJONG (2007), a formação de estruturas reprodutivas está diretamente relacionada com a posição das mesmas em relação a exposição à luz durante o ciclo anterior, sendo que quanto maior a área exposta a luz, maior é a formação destas estruturas.

Tabela 1. Frutificação efetiva de pereiras europeias submetidas à tratamentos de controle de vigor, durante a safra 2012/2013, no município de Vacaria, RS.

Table 1. Fruit set of European pear trees submitted to growth control treatments, during the 2012/2013 season, in Vacaria city, RS, Brazil.

\begin{tabular}{lccccc}
\hline \multicolumn{1}{c}{ Tratamento/Cultivar } & Packham's & William's & Abate Fetel & Red Bartlett & Carrick \\
\hline Testemunha & $11 \mathrm{~ns}$ & $11 \mathrm{~ns}$ & $1 \mathrm{~ns}$ & $7 \mathrm{~ns}$ & $3 \mathrm{~ns}$ \\
Anelamento simples & 10 & 8 & 1 & 10 & 3 \\
Anelamento duplo & 12 & 10 & 2 & 9 & 5 \\
Corte simples & 8 & 11 & 1 & 8 & 7 \\
Corte duplo & 12 & 9 & 1 & 7 & 3 \\
Trin-etil 1600 mg L-1 & 17 & 10 & 128 & 12 & 2 \\
\hline CV (\%) & 14 & 14 & & & 47 \\
\hline ns-não significativo & & & & & \\
\hline
\end{tabular}

ns - não significativo.

A produtividade da cultivar Abate Fetel no ciclo 2012/13 foi baixa e desuniforme, em todos os tratamentos, não sendo alterada por efeito destes. Além da baixa frutificação efetiva, esta cultivar apresentou baixas produtividades. A pereira 'Carrick' não apresentou diferença da produtividade das plantas submetidas aos diferentes tratamentos, variando de 10 a $16 \mathrm{t} \mathrm{ha}^{-1}$ (Tabela 2).

Não houve efeito dos tratamentos na produtividade da cultivar William's e de seu clone 'Red Bartlett', as quais apresentaram produtividades elevadas de 54 e $42 \mathrm{t} \mathrm{ha}^{-1}$, respectivamente. RAFFO et al. (2011), 
utilizando anelamento (6 - $8 \mathrm{~mm}$ ), não encontraram nenhum efeito significativo sobre produtividade ou tamanho dos frutos em pereiras 'William's' aneladas 20 dias após a plena floração. Embora o anelamento possa retardar o crescimento vegetativo, seu efeito ainda é muito variável devido à interferência de fatores como cultivar, idade da planta, vigor, portaenxerto, tipo de solo, entre outros (SMIT et al. 2005).

A cultivar Packham's Triumph apresentou as plantas mais produtivas, em média $86 \mathrm{t} \mathrm{ha}^{-1}$. A aplicação de Trin-etil reduziu a produtividade das pereiras quando comparadas aos demais tratamentos (Tabela 2). Por ser um produto que atua reduzindo a síntese de giberelinas (RADEMACHER 2014), a redução de produtividade pode estar relacionada à redução dos níveis deste hormônio na planta, já que este atua no processo de frutificação e desenvolvimento de frutos (TAIZ \& ZEIGER 2013).

Tabela 2. Produtividade estimada de pereiras europeias submetidas à tratamentos de controle de vigor, durante a safra 2012/2013, no município de Vacaria, RS.

Table 2. Estimated productivity of European pear trees submitted to growth control treatments, during the 2012/2013 season, in Vacaria city, RS, Brazil.

\begin{tabular}{llllll}
\hline \multicolumn{5}{c}{ Produtividade estimada (t ha ${ }^{-1}$ ) } \\
\hline \multicolumn{1}{c}{ Tratamento/Cultivar } & Packham's & William's & Abate Fetel & Red Bartlett & Carrick \\
\hline Testemunha & $85 \mathrm{a}$ & $57 \mathrm{~ns}$ & $3 \mathrm{~ns}$ & $41 \mathrm{~ns}$ & $19 \mathrm{~ns}$ \\
Anelamento simples & $88 \mathrm{a}$ & 51 & 7 & 43 & 26 \\
Anelamento duplo & $81 \mathrm{a}$ & 54 & 1 & 38 & 23 \\
Corte simples & $97 \mathrm{a}$ & 56 & 5 & 39 & 17 \\
Corte duplo & $89 \mathrm{a}$ & 49 & 4 & 44 & 10 \\
Trin-etil 1600 mg L $^{-1}$ & $75 \mathrm{~b}$ & 57 & 5 & 46 & 26 \\
\hline CV (\%) & 15 & 15 & 127 & 28 & 66 \\
\hline
\end{tabular}

Médias seguidas de mesma letra na coluna não diferem entre si pelo teste Scott-knott à $5 \%$ de probabilidade de erro. ns - não significativo.

Em relação ao diâmetro de frutos, considerou-se pequenos os frutos abaixo de $65 \mathrm{~mm}$ de diâmetro, médios entre 65 e $80 \mathrm{~mm}$ e grande quando maiores de $80 \mathrm{~mm}$. Não houve diferença significativa entre os tratamentos para esta característica (dados não apresentados). Verificou-se no primeiro ano em estudo, que as pereiras Abate Fetel, as quais tiveram baixa produtividade, apresentaram em torno de $60 \%$ dos frutos de tamanho médio. Já a cultivar 'Carrick', em torno da metade dos frutos enquadravam-se na classe de frutos pequenos e a outra metade nos frutos médios. Cerca de $75 \%$ dos frutos das pereiras 'William's' e 'Red Bartlett' enquadraram-se na classificação dos frutos pequenos, com o restante na classe de frutos médios. A elevada porcentagem de frutos classificados como pequenos é resultado do elevado número de frutos por planta, pois ocorre competição entre si pelos fotoassimilados, o que reduz o tamanho (GREENE 2008).

Em contrapartida, a pereira 'Packham's', a qual foi a mais produtiva dentre as estudadas, produziu a maior parte dos frutos (51\%) pertencentes à classe de frutos médios $(65-80 \mathrm{~mm})$, seguidos pela classe dos menores que $65 \mathrm{~mm}$ (37\%) e maiores que $80 \mathrm{~mm}$ (11\%). Mesmo com o excesso de produção, apenas $37 \%$ dos frutos foram classificados como pequenos. Isto se deve à característica genética desta cultivar em produzir frutos grandes (MACHADO et al. 2012).

A avaliação de tratamentos redutores de crescimento é complicada, devido à falta de um único e simples método para medir com precisão o que caracteriza crescimento vegetativo das plantas (FORSHEY 1989, FORSHEY \& ELFVING 1989). Uma forma comumente utilizada para avaliação do vigor de plantas é através da mensuração do comprimento médio dos ramos do ano e também do crescimento anual da copa, através da multiplicação do comprimento médio pelo número de ramos do ano de cada planta (Tabela 3).

Em relação ao comprimento médio dos ramos do ano, verificou-se diferença estatística apenas na cultivar Abate Fetel, onde houve redução no comprimento médio dos ramos das pereiras submetidas aos cortes de tronco e também ao anelamento duplo. Já no crescimento médio anual da copa, houve redução do crescimento da cultivar Packham's Triumph submetida aos tratamentos de anelamento duplo (37\%), corte de tronco simples (24\%) e aplicação de Trin-etil $1600 \mathrm{mg} \mathrm{L}^{-1}(31 \%)$. As demais cultivares não apresentaram diferenças entre os tratamentos. A cultivar Packham's apresentou o maior crescimento anual da copa, em média $69 \mathrm{~m}$ planta $^{-1}$, enquanto que a cultivar Abate Fetel apresentou o menor crescimento, em média 16 m planta $^{-1}$.

DREYER (2013) descreve a ineficiência da redução do crescimento vegetativo das pereiras 'Abate Fetel' e 'Forelle' submetidas ao anelamento $(3 \mathrm{~mm})$ em plena floração, atribuindo o resultado à pouca área de casca retirada pelo anelamento.

O efeito dos tratamentos em relação às características físico-químicas dos frutos foi diferente entre as cultivares, como pode ser observado na Tabela 4. As técnicas de corte de tronco e aplicação de Trin-etil 
reduziram a firmeza de polpa dos frutos de 'Abate Fetel'. Nos frutos das demais cultivares não houve diferenças na firmeza de polpa.

Tabela 3. Comprimento médio de ramos do ano $(\mathrm{cm})$ e crescimento médio anual ( $\mathrm{m}$ planta-1) das pereiras europeias submetidas à tratamentos de redução de vigor, na safra 2012/2013, Vacaria, RS.

Table 3. Average shoot length $(\mathrm{cm})$ and annual shoot growth $(\mathrm{m}$ planta-1) of European pear trees submitted to growth control treatments, during the 2012/2013 season, in Vacaria city, RS, Brazil.

\begin{tabular}{|c|c|c|c|c|c|c|}
\hline \multicolumn{7}{|c|}{ Comprimento médio do ramo do ano $(\mathrm{cm})$} \\
\hline & Packham's & William's & Aba & te Fetel & Red Bartlett & Carrick \\
\hline Testemunha & $29 \mathrm{~ns}$ & $35 \mathrm{~ns}$ & 16 & $\mathrm{~b}$ & $26 \mathrm{~ns}$ & $21 \mathrm{~ns}$ \\
\hline Anelamento simples & 31 & 33 & 15 & $\mathrm{~b}$ & 27 & 21 \\
\hline Anelamento duplo & 25 & 32 & 14 & a & 24 & 22 \\
\hline Corte simples & 26 & 31 & 14 & a & 22 & 21 \\
\hline Corte duplo & 28 & 31 & 14 & a & 28 & 21 \\
\hline Trin-etil $1600 \mathrm{mg} \mathrm{L}^{-1}$ & 27 & 31 & 17 & $\mathrm{~b}$ & 25 & 24 \\
\hline $\mathrm{CV}(\%)$ & 18 & 13 & 11 & & 12 & 26 \\
\hline \multicolumn{7}{|c|}{ Crescimento médio anual (m planta $\left.{ }^{-1}\right)$} \\
\hline & Packham's & William's & Aba & te Fetel & Red Bartlett & Carrick \\
\hline Testemunha & $82 \mathrm{a}$ & $38 \mathrm{~ns}$ & $15 \mathrm{~ns}$ & & $33 \mathrm{~ns}$ & $39 \mathrm{~ns}$ \\
\hline Anelamento simples & $81 \mathrm{a}$ & 44 & 20 & & 37 & 38 \\
\hline Anelamento duplo & $51 \mathrm{~b}$ & 39 & 15 & & 35 & 39 \\
\hline Corte simples & $62 \mathrm{~b}$ & 44 & 13 & & 29 & 35 \\
\hline Corte duplo & $72 \mathrm{a}$ & 37 & 12 & & 38 & 40 \\
\hline Trin-etil $1600 \mathrm{mg} \mathrm{L}^{-1}$ & $56 \mathrm{~b}$ & 33 & 21 & & 26 & 47 \\
\hline CV (\%) & 15 & 26 & 25 & & 22 & 24 \\
\hline
\end{tabular}

Médias seguidas de mesma letra na coluna não diferem entre si pelo teste Scott-knott à $5 \%$ de probabilidade de erro. ns - não significativo.

A técnica de corte de tronco simples e a aplicação de Trin-etil reduziram a concentração de sólidos solúveis dos frutos da cultivar William's. Já na cultivar Red Bartlett, o corte de tronco simples aumentou a concentração de sólidos solúveis dos frutos, bem como o anelamento duplo. Nos frutos das demais cultivares não houve diferenças na concentração de sólidos solúveis.

Tabela 4. Firmeza de polpa, sólidos solúveis e acidez titulável de frutos de pereiras europeias submetidas à tratamentos de redução de vigor, na safra 2012/2013 em Vacaria, RS.

Table 4. Flesh firmness, soluble solids and titratable acidity of European pear fruits submitted to growth control treatments, during the 2012/2013 season, in Vacaria city, RS, Brazil.

\begin{tabular}{|c|c|c|c|c|c|c|c|c|c|c|}
\hline Cultivares & Variável/Tratamento & Test. & $\begin{array}{c}\text { Anel. } \\
\text { Simples }\end{array}$ & $\begin{array}{l}\text { Anel. } \\
\text { Duplo }\end{array}$ & $\begin{array}{c}\text { Corte } \\
\text { Simples }\end{array}$ & $\begin{array}{l}\text { Corte } \\
\text { Duplo }\end{array}$ & $\begin{array}{c}\text { Moddus } \AA \\
1600 \\
\mathrm{mg} \mathrm{L}^{-1}\end{array}$ & $\begin{array}{c}\text { STRG } \\
100 \mathrm{mg} \\
\mathrm{L}^{-1}\end{array}$ & & $\begin{array}{l}\text { CV } \\
(\%)\end{array}$ \\
\hline \multirow{3}{*}{ Packham's } & Firmeza (libras $\mathrm{cm}^{-2}$ ) & 14,7 a & 14,7 a & 14,6 a & 14,4 a & 14,5 a & 14,9 a & 13,1 & $b$ & 4,5 \\
\hline & Sólidos Sol. (ํㅏrix) & $11,6 \mathrm{~ns}$ & 11,4 & 12,3 & 11,5 & 11,3 & 10,7 & 11,7 & & 4,8 \\
\hline & Acidez Tit. $\left(\mathrm{meq} \mathrm{L}^{-1}\right)$ & $24,3 \mathrm{~ns}$ & 20,9 & 21,2 & 21,5 & 20,8 & 23,2 & 20,6 & & 11,7 \\
\hline \multirow{3}{*}{ William's } & Firmeza (libras $\mathrm{cm}^{-2}$ ) & $15,5 \mathrm{~ns}$ & 15,0 & 14,8 & 15,3 & 15,7 & 15,3 & 15,5 & & 4,9 \\
\hline & Sólidos & $10,2 \mathrm{a}$ & $10,6 \mathrm{a}$ & 10,3 a & $10,1 \mathrm{~b}$ & $10,5 \mathrm{a}$ & $9,8 \quad b$ & 10,5 & a & 3,5 \\
\hline & Acidez Tit. ( & $35,1 \mathrm{~ns}$ & 32,6 & 34,1 & 33,1 & 35,9 & 33,9 & 30,7 & & 10,4 \\
\hline \multirow{3}{*}{ Abate Fetel } & Firmeza (libras & 11,9 a & 11,7 a & $11,6 \mathrm{a}$ & $10,3 b$ & $10,7 b$ & $11,1 \mathrm{~b}$ & 10,8 & $b$ & 7,3 \\
\hline & Sólidos Sol. ("obrix) & $13,1 \mathrm{~ns}$ & 13,0 & 13,0 & 13,0 & 13,0 & 13,3 & 13,4 & & 4,5 \\
\hline & Acidez Tit. & $30,1 \mathrm{~b}$ & 31,8 a & 34,2 a & $29,1 \quad b$ & $28,4 \mathrm{~b}$ & $28,9 \mathrm{~b}$ & 27,9 & $b$ & 9,2 \\
\hline \multirow{3}{*}{ Red Bartlett } & Firm & $12,6 \mathrm{~ns}$ & 13,1 & 12,1 & 12,3 & 13,5 & 12,5 & 11,6 & & 9,8 \\
\hline & Sólidos Sol. (ํㅏix) & $11,0 \mathrm{~b}$ & $10,8 \mathrm{~b}$ & 11,3 a & 11,3 a & $10,8 \mathrm{~b}$ & $10,9 \mathrm{~b}$ & 11,6 & a & 3,4 \\
\hline & Acidez Tit. (meq L-1) & $23,4 \mathrm{~ns}$ & 24,2 & 21,6 & 22,6 & 23,3 & 23,7 & 20,3 & & 10,3 \\
\hline \multirow{3}{*}{ Carrick } & Firmeza (libras $\mathrm{cm}^{-2}$ ) & $12,8 \mathrm{~ns}$ & 12,4 & 12,7 & 12,3 & 12,2 & 13,0 & 12,3 & & 4,6 \\
\hline & Sólidos Sol. (ํrix) & $13,6 \mathrm{~ns}$ & 13,7 & 13,5 & 14,3 & 14,1 & 13,9 & 13,8 & & 3,3 \\
\hline & Acidez Tit. (meq $\mathrm{L}^{-1}$ ) & 68,7 a & $68,5 \mathrm{~b}$ & $65,1 \mathrm{~b}$ & $71,3 \mathrm{a}$ & 73,9 a & $65,2 \mathrm{~b}$ & 69,0 & $a$ & 6,8 \\
\hline
\end{tabular}

Médias seguidas de mesma letra na linha não diferem entre si pelo teste Scott-knott à $5 \%$ de probabilidade de erro. ns - não significativo. 
Apenas as cultivares Abate Fetel e Carrick apresentaram diferenças na acidez dos frutos, sendo que os anelamentos aumentaram a acidez dos frutos de 'Abate Fetel', enquanto que estes mesmos tratamentos, juntamente com a aplicação de Trin-etil reduziram a acidez dos frutos da pereira 'Carrick'.

As pereiras 'Packham's', 'William's' e 'Red Bartlett' apresentaram alternância de produção onde na safra 2012/13 obtiveram elevadas produtividades e na safra seguinte não apresentaram florescimento. De acordo com GREENE (2008), a elevada produção de frutos eleva a concentração de giberelinas produzidas pelas sementes, juntamente com o esgotamento de carboidratos na planta sendo as principais causas da alternância de produção.

Embora não quantificado, a alternância de produção pode ter sido intensificada pela desfolha precoce que ocorreu logo após a colheita, ocasionada pela infecção de entomosporiose. Já as cultivares Abate Fetel e Carrick, as quais produziram poucos frutos, obtiveram incrementos no florescimento e na frutificação efetiva na safra seguinte.

A aplicação de Trin-etil reduziu o retorno da floração das pereiras 'Abate Fetel' e 'Carrick' em comparação às plantas testemunhas. Por outro lado, o retorno de floração da pereira 'Abate Fetel' foi incrementado pela prática de anelamento e corte de tronco duplo. Já na cultivar Carrick, houve incremento do retorno de floração nas plantas submetidas aos tratamentos de anelamento duplo e corte de tronco, independente da intensidade, quando comparado às plantas testemunhas (Tabela 5).

Tabela 5. Frutificação efetiva e retorno de floração das pereiras Abate Fetel e Carrick submetidas à tratamentos de redução de vigor, na safra 2013/2014 em Vacaria, RS.

Table 5. Fruit set and return bloom of 'Abate Fetel' and 'Carrick' pear trees submitted to growth control treatments, during the 2013/2014 season, in Vacaria city, RS, Brazil.

\begin{tabular}{|c|c|c|c|c|}
\hline & \multicolumn{2}{|c|}{ Abate Fetel } & \multicolumn{2}{|r|}{ Carrick } \\
\hline & $\%$ frut. efetiva & $\mathrm{n}^{\circ}$ de flores planta ${ }^{-1}$ & $\%$ frut. efetiva & $\mathrm{n}^{\circ}$ de flores planta ${ }^{-1}$ \\
\hline Testemunha & $0,0 \quad b$ & $831 \mathrm{~b}$ & 5 & $743 \mathrm{~b}$ \\
\hline Anelamento simples & 0,5 a & 1152 a & $16 \mathrm{a}$ & 952 b \\
\hline Anelamento duplo & $1,0 \quad a$ & $1005 \mathrm{a}$ & $8 \mathrm{~b}$ & 1640 a \\
\hline Corte simples & $1,1 \quad \mathrm{a}$ & 899 b & 8 & 1127 \\
\hline Corte duplo & $0,4 \quad a$ & 1126 a & 7 & 1470 \\
\hline Trin-etil $1600 \mathrm{mg} \mathrm{L}^{-1}$ & $0,0 \mathrm{~b}$ & $495 \mathrm{c}$ & $17 \mathrm{a}$ & $404 \mathrm{c}$ \\
\hline CV (\%) & 70 & 14 & 7 & 22 \\
\hline
\end{tabular}

Médias seguidas de mesma letra na coluna não diferem entre si pelo teste Scott-knott à $5 \%$ de probabilidade de erro.

Tabela 6. Produtividade $\left(\mathrm{t} \mathrm{ha}^{-1}\right)$ e classes de diâmetro $(\mathrm{mm})$ de frutos das pereiras 'Abate Fetel' e 'Carrick' submetidas à tratamentos de redução de vigor, na safra 2013/2014, Vacaria, RS.

Table 6. Yield $\left(t \mathrm{ha}^{-1}\right)$ and fruit diameter classes $(\mathrm{mm})$ of 'Abate Fetel' and 'Carrick' pear trees submitted to growth control treatments, during the 2013/2014 season, in Vacaria city, RS, Brazil.

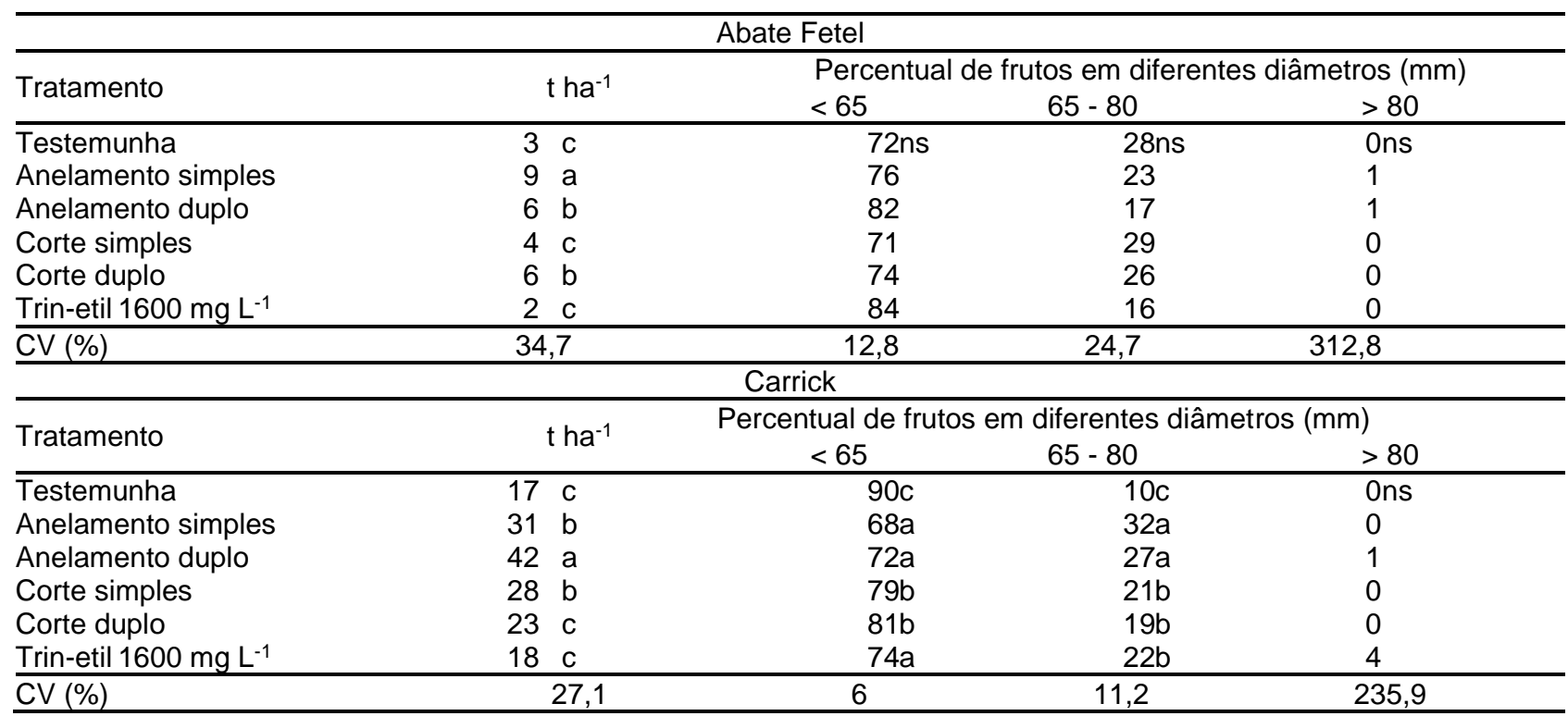

Médias seguidas de mesma letra na coluna não diferem entre si pelo teste Scott-knott à $5 \%$ de probabilidade de erro.

ns - não significativo. 
As técnicas de anelamento e corte de tronco aumentaram a frutificação efetiva da cultivar Abate Fetel. Já na cultivar Carrick, todos os tratamentos aumentaram a frutificação efetiva, com destaque para o anelamento simples e o corte de tronco duplo, os quais triplicaram a frutificação efetiva quando comparado com as plantas testemunhas (Tabela 5). Resultados similares foram encontrados por DREYER (2013), onde houve aumento do retorno de floração da cultivar Abate Fetel, sem ter efeito sobre o crescimento vegetativo.

A produtividade das pereiras 'Abate Fetel' submetidas ao anelamento simples triplicou (Tabela 6). A utilização do anelamento duplo e corte de tronco duplo também aumentaram a produtividade desta cultivar, no entanto, ainda é uma produtividade baixa, sendo atribuída à deficiência de polinização, pois não haviam outras cultivares de pereira coincidindo florescimento, bem como verificou-se baixo número de sementes nos frutos fixados.

Por outro lado, as pereiras 'Carrick' submetidas ao anelamento duplo obtiveram um incremento na produtividade de $147 \%$ comparado com as plantas testemunhas (Tabela 6). As pereiras submetidas ao anelamento e corte de tronco simples apresentaram produtividade superior às testemunhas de $82 \%$ e $65 \%$, respectivamente. Além disso, todos os tratamentos reduziram a porcentagem de frutos pequenos (menores que $65 \mathrm{~mm}$ ) e aumentaram a quantidade de frutos médios (entre 65 e $80 \mathrm{~mm}$ ), com destaque para as duas intensidades de anelamento (Tabela 6). De acordo com THERON \& STEYN (2008), estas técnicas mudam a relação fonte/dreno de carboidratos, em que os frutos passam a ser drenos principais possibilitando seu maior crescimento.

\section{CONCLUSÃO}

Anelamento duplo, corte de tronco simples e aplicação de Trinexapac-etil (1600 $\mathrm{mg} \mathrm{L}^{-1}$ ) reduzem o crescimento vegetativo da pereira Packham's Triumph, mas não reduzem o crescimento vegetativo das cultivares William's, Red Bartlett, Abate Fetel e Carrick.

A utilização da técnica de anelamento e corte de tronco incrementam o retorno de floração e a produtividade das pereiras 'Abate Fetel' e 'Carrick'.

A utilização de Trinexapac-etil (1600 $\mathrm{mg} \mathrm{L}^{-1}$ ) em pereiras 'Abate Fetel' e 'Carrick' reduz o retorno de florescimento no ciclo seguinte à sua aplicação.

\section{AGRADECIMENTOS}

Os autores agradecem à CAPES pelo auxílio financeiro e concessão de bolsas e ao Sr. Eng. Agr. Genor Mussatto por ter cedido seu pomar como área experimental.

\section{REFERÊNCIAS}

CAIN JC. 1971. Effects of mechanical pruning of apple hedgerows with a slotting saw on light penetration and fruiting. Journal of the America Society for Horticultural Science 96: 664-667.

DECKERS T et al. 2005. Natural or chemical growth regulation in pear. Acta Horticulturae 671: 503-516.

DEJONG TM. 2007. Canopy and light management. IN: MITCHAM EJ \& ELKINS RB. (Eds.). Pear Production and Handling Manual. Oakland: University of California. Agriculture and Natural Resourses. p. 59-62.

DREYER C. 2013. Fruit set and fruit size studies on 'Forelle' and 'Abate Fetel' pear (Pyrus communis L.). Thesis (Master), Faculty of Agricultural Sciences, Stellenbosch University, Stellenbosch: South Africa. $108 \mathrm{f}$.

FERREIRA DF. 2010. Programa computacional Sisvar. Versão 5.3. Lavras: UFLA.

FORSHEY CG. 1989. Measuring the effects of growth regulators on the vegetative growth-fruiting relationship in apple trees. Acta Horticulturae 239: 211-220.

FORSHEY CG \& ELFVING DC. 1989. The relationship between vegetative growth and fruiting in apple trees. Horticultural Reviews 11: 229-287.

GREENE DW. 2008. The effects of repeat annual applications of prohexadione-calcium on fruit set, return bloom, and fruit size of apples. Horticultural Science 43: 376-379.

IUCHI T \& LUZ AR. 2010. Crescimento, frutificação, poda e condução da pereira. In: III Reunião Técnica da Cultura da Pereira: Busca pela identidade nacional. Anais... Lages: UDESC. p. 96-101.

IUCHI T et al. 2008. Anelamento e paclobutrazol na produção e absorção de nutrientes em pereira (Pyrus communis L.) cultivar Packham's Triumph. Revista Brasileira de Fruticultura 30: 857-861.

JACKSON JE. 2003. Biology of apples and pears. Cambridge: University Press. 488p.

LUZ AR et al. 2012. Floração e polinização. In: RUFATO L et al. A cultura da Pereira. p. 38-53.

MACHADO BD et al. 2012. Cultivares de pereiras europeias. In: RUFATO L et al. A cultura da Pereira, p.150-171.

QUEZADA AC et al. 2003. Pêra: produção. Embrapa clima temperado. Embrapa Informação Tecnológica. 105p.

RADEMACHER W. 2014. Prohexadione-Ca and Trinexapac-Ethyl: Similarities in Structure but Differences in Biological Action. Acta Horticulturae 1042: 33-41.

RAFFO MD et al. 2011. Effect of trunk girdling on fruit production, fruit size and tree vigor on "Bartlett" pears in Rio Negro 
and Neuquén valley, Argentina. Acta Horticulturae 909: 645-650.

SMIT M et al. 2005. Shoot growth control of pear trees (Pyrus communis L.) with prohexadione-calcium. Scientia Horticulturae 106: 515-529.

TAIZ L \& ZEIGER E. 2013. Fisiologia vegetal. 5.ed. Porto Alegre: Artmed. 954p.

THERON KI \& STEYN WJ. 2008. Girdling: science behind the age-old technique. Acta Horticulturae 800: 51-60.

VAN DER ZWET T \& CHILDERS NF. 1982. The Pears, Cultivars to Marketing. Gainesville, Florida, EUA, 502p. 